\title{
Étendre les frontières de la coordination pour soigner les migrants. L'exemple d'un réseau de santé précarité en Île-de-France
}

Isabelle Siffert

\section{(2) OpenEdition}

1 Journals

Édition électronique

URL : https://journals.openedition.org/rfst/532

DOI : $10.4000 /$ rfst.532

ISSN : 2492-3672

Traduction(s) :

Breaking healthcare coordination boundaries in response to migrants precariousness: the case study of a healthcare network in the Parisian suburbs - URL : https://journals.openedition.org/rfst/800 [en]

Éditeur

Espaces et SOciétés (UMR 6590)

Référence électronique

Isabelle Siffert, «Étendre les frontières de la coordination pour soigner les migrants. L'exemple d'un réseau de santé précarité en Île-de-France », Revue francophone sur la santé et les territoires [En ligne], Miscellanées, mis en ligne le 17 décembre 2020, consulté le 03 mai 2021. URL : http:// journals.openedition.org/rfst/532; DOI : https://doi.org/10.4000/rfst.532

Ce document a été généré automatiquement le 3 mai 2021.

\section{(c) (i) (2)(2)}

La Revue francophone sur la santé et les territoires est mise à disposition selon les termes de la Licence Creative Commons Attribution - Pas d'Utilisation Commerciale - Partage dans les Mêmes Conditions 4.0 International. 


\title{
Étendre les frontières de la coordination pour soigner les migrants. L'exemple d'un réseau de santé précarité en Île-de-France
}

\author{
Isabelle Siffert
}

\section{Introduction}

1 L'étude des relations entre santé et migration connaît une multiplication de travaux de recherche en sciences sociales depuis les années 1990. Rejetant des approches exotiques, hygiénistes ou encore culturalistes, ils ont d'abord dénoncé la cécité du système de soins face aux inégalités sociales de santé liées aux origines (Fassin et al., 2000) et n'ont cessé d'interroger l'existence d'une santé particulière des populations étrangères. L'expression "santé des migrants ", à la fois controversée et de plus en plus usitée, désigne à la fois l'évolution de l'état de santé au cours de l'expérience migratoire, l'accès aux droits et au système de santé au cours et à l'issu de la migration et la prise en charge des personnes migrantes dans les services de santé. Derrière l'idée d'un état de santé et de pratiques de soins plus ou moins spécifiques, l'expression renvoie alors aux représentations de la figure du «migrant » et de son corps dans les lieux d'accueil et de soin. Le terme « migrant » désigne une personne ayant quitté son pays d'origine pour s'installer dans un autre, sans distinction sur le pays d'origine ou la situation économique et sociale. En pratique, le terme est massivement repris dans les discours et médias pour désigner les populations fuyant leurs pays touchés par des crises géopolitiques et économiques. Il est alors largement associé à la figure de l'étranger précaire, occupant de campement ou hébergé en situation instable, demandeur d'asile ou en situation irrégulière sur le territoire. Derrière le mot "migrant", bien plus que celui d'«immigré", se cache une condition d'éternel nomade, qui s'avère être un frein à l'idée d'un accueil durable sur le territoire (Agier, 2016). Il masque alors les motifs de migration et les situations administratives 
extrêmement variables dans lesquelles se trouvent les individus désignés. Certains arrivent sur le territoire avec un titre de séjour provisoire, d'autres sans. Parmi eux, les demandeurs d'asile désignent ceux qui font une demande de protection internationale au titre de la Convention de Genève. Ils reçoivent un récépissé de demande d'asile, garant de leur situation régulière provisoire sur le territoire français. A l'issue de la décision de l'Office de protection des réfugiés et des apatrides (OFPRA), les demandeurs d'asile peuvent obtenir le statut de réfugié ou la protection subsidiaire donnant lieu à une carte de séjour. A l'inverse, ceux qui se voient refuser le droit d'asile sont dits " déboutés » et se retrouvent alors " sans-papiers », c'est-à-dire en situation irrégulière sur le territoire français.

2 Ces différences de statut, conjuguées à des conditions de vie très variables, déterminent des différences dans l'accès aux droits et au système de santé. Des travaux mêlant des approches épidémiologiques et sociologiques ont notamment montré des prévalences particulièrement élevées pour certaines pathologies, comme le VIH, les hépatites, la tuberculose mais aussi le diabète et certaines maladies cardio-vasculaires (Mizrahi et al, 1993 ; Lot et al, 2012 ; Drouot et al, 2012, Desgrées du Loû, 2017). De façon plus générale est décrit un accès aux soins tardif entraînant des retards de diagnostic et de prise en charge, et une dégradation de l'état de santé après l'arrivée sur le territoire français. Cette vulnérabilité, à la fois dans l'exposition au risque et dans l'évolution des maladies, est en large partie inscrite dans des conditions de vie et de soins, marquées par un accès insuffisant et retardé à la prévention, aux soins primaires et aux traitements. Des travaux ont alors analysé comment l'accès aux services de santé par les étrangers en situation précaire évolue entre un décloisonnement du système de santé et le développement de programmes spécifiques (Comiti \& Patureau, 2005 ; Chambon \& Le Goff, 2016). A l'ambition juridique d'un accès universel aux soins s'oppose une réalité marquée par des barrières sociales et administratives à l'obtention d'une couverture maladie (Carde, 2009; Maille \& Toullier, 2009; Chappuis, 2012). Par conséquent, les réseaux d'acteurs mobilisés par les populations en situation de précarité indiquent des difficultés de plus en plus importantes à recourir au système de santé dit « de droit commun " pour les étrangers en situation précaire et leur surreprésentation dans les dispositifs de soins gratuits dédiés à ceux qui ne peuvent (encore) prétendre à la protection universelle maladie (Mizrahi \& Mizrahi, 2000 ; Parizot \& Chauvin, 2003 ; Médecins du Monde, 2018).

3 Si les mécanismes à l'origine des inégalités de santé liées à l'expérience migratoire commencent à être mieux connus (Fassin et al., 2000 ; Carde, 2007; Berchet \&Jusot, 2010 ; Cognet et al, 2012), ces dispositifs de soins accueillant des populations sans couverture sociale, leur organisation et la coordination que suppose l'accueil de populations étrangères sans ressources, ont peu été décrits. C'est alors dans cette démarche que s'inscrit cet article.

4 Sans les opposer, on peut distinguer deux catégories de dispositifs de soins gratuits : d'un côté les dispositifs publics, produits des politiques sanitaires et sociales; de l'autre, les structures associatives, qui au contraire interrogent le désengagement de l'Etat dans la lutte contre les inégalités sociales. Les principales ressources publiques dédiées aux personnes exclues du système de santé de droit commun sont les Permanences d'accès aux soins de santé (PASS) et les Equipes mobiles psychiatrie précarité (EMPP). Les PASS sont des services majoritairement hospitaliers, créés en 1998 par la loi de lutte contre les exclusions, définis comme des lieux d'accueil et de 
soins destinés aux personnes sans couverture sociale ${ }^{1}$. Il existe une importante variabilité des services PASS, tant dans leur fonctionnement, leurs activités et leur intégration dans des réseaux plus larges, mais sont des dispositifs de plus en plus visibles et relativement bien répertoriés. Elles sont rapidement devenues les principales références de l'accès aux soins des publics précaires (Platon \& Lamouche, 2012) comme en témoigne la multiplication de travaux sur la prise en charge des migrants en leur sein (Geeraert, 2017; Georges-Tarragano et al, 2017; Tapié de Céleyran et al, 2017). Les EMPP, mises en place en 2005 dans le cadre du développement des programmes régionaux de l'accès à la prévention et aux soins (PRAPS) ${ }^{2}$, sont des dispositifs rattachés à des services hospitaliers de psychiatrie visant à « aller-vers » les personnes en situation d'exclusion sociale via des maraudes et des interventions auprès de structures d'accueil social. Les EMPP sont des dispositifs encore émergents, elles font alors peu l'objet de travaux de recherche en dehors des revues spécialisées en psychiatrie qui en décrivent le fonctionnement et les évolutions (Marques, 2013).

5 Pour la plupart antérieures aux PASS et aux EMPP, les structures associatives sont le fruit d'initiatives plus ou moins locales en réponse à des situations sanitaires et sociales. Ces initiatives s'inscrivent dans des contextes locaux variés et existent sous différentes formes : on retrouve notamment des antennes locales d'ONG, des réseaux de santé (Hervé, 2007) ou encore des associations spécialisées dans l'accueil de populations exilées. Si elles peuvent être financées en partie par des fonds publics (des dotations des Agences régionales de santé, des subventions communales ou départementales), leur statut associatif leur permet d'être porteuses de discours et d'actions militantes et de s'appuyer sur des bénévoles. Des travaux ont décrit le rôle majeur de ces initiatives associatives dans la prise en charge des migrants tout en soulignant la diversité de leurs modes d'action selon les contextes territoriaux dans lesquelles elles s'inscrivent (Hoyez, 2011 ; D'Halluin, 2012 ; D'Halluin \& Hoyez, 2012). Elles restent néanmoins bien moins connues et décrites que les dispositifs hospitaliers et sont plus difficilement identifiées par les usagers, alors même qu'elles ont souvent été précurseurs dans la lutte contre l'exclusion sur leur territoire.

6 Le contexte d'intensification des flux migratoires vers l'Europe et la cristallisation d'une « crise de l'accueil des migrants » depuis $2015^{3}$ (Beauchemin \& Ichou, 2016) ont contribué à accélérer la constitution de la santé des migrants comme problématique scientifique, sociale et politique. Cela s'est traduit notamment par la multiplication de travaux de recherche sur leur accueil dans des dispositifs spécifiques et la profusion de prérogatives nationales sur les modalités et moyens de leur prise en charge à différentes échelles. En juin 2018, une circulaire du Ministère des solidarités et de la santé encourage la mise en place de " parcours de santé des migrants »" Reconnaissant que la prise en charge médico-sociale des étrangers primo-arrivants repose principalement sur les dispositifs dédiés aux populations exclues du système de santé de droit commun, elle confirme une perspective de mobiliser davantage les acteurs médico-sociaux de proximité en passant par la mise en place de supports de coordination: espace de travail partagé, répertoire des structures ressources du territoire, cartographie des services, conventions de partenariat, structure " pivot ». De l'accès à la prévention à la prise en charge des psycho-traumatismes liés à l'exil, elle invite à réfléchir aux moyens mis à disposition des acteurs pour se constituer en réseau et coordonner l'accueil de populations migrantes. 
7 Le présent article s'intéresse à un territoire où les premières initiatives en matière d'accès aux soins des plus démunis, et notamment des populations migrantes, ont été portées par un réseau de santé associatif. A partir de l'étude de ce réseau installé sur un territoire de grande couronne francilienne, il propose de réfléchir aux évolutions de la prise en charge médico-sociale des étrangers en situation précaire, en particulier demandeurs d'asile, et aux enjeux de coordination qui y sont associés. Il analyse le déploiement d'une "problématique migrants » entre les acteurs médicaux et sociaux d'un territoire et l'évolution de leurs activités et partenaires dans le contexte d'un accueil de plus en plus important de populations étrangères en situation précaire. Alors que les prérogatives incitent à la formalisation de parcours de soins, il s'agit de porter un regard sur les ressources et contraintes de coordination des acteurs engagés dans leur accueil. La première partie présente les activités du réseau de santé et de ses deux principaux partenaires, la PASS et l'EMPP de secteur. En s'attardant sur son histoire et l'évolution de sa file active, elle vise à replacer les activités du réseau dans le contexte migratoire local et caractériser la visibilité auprès de ses partenaires. La seconde partie analyse le développement de dispositifs et modes d'actions relevant d'une spécification de la prise en charge des populations migrantes précaires. Elle revient alors sur les difficultés liées à la prise en compte de l'instabilité résidentielle des populations. Face à cela, la dernière partie interroge les capacités d'étendre la coordination de ces acteurs médico-sociaux, dans un contexte d'insuffisance des moyens et technologies adaptés.

\section{Méthodologie de la recherche}

Ce travail s'inscrit dans une recherche doctorale sur l'accès aux soins des personnes en demande d'asile sur un territoire de la grande couronne francilienne. Il s'appuie en particulier sur une étude de terrain menée entre mars et juillet 2019 auprès d'un réseau ville-hôpital (RVH) et ses deux principaux partenaires, l'équipe mobile psychiatrie précarité (EMPP) et la permanence d'accès aux soins de santé (PASS) de l'hôpital de secteur, installés en Île-de-France, à environ $50 \mathrm{~km}$ de Paris. Pour les trois structures, j'ai d'abord effectué de l'observation en immersion dans les équipes, en les accompagnant dans leurs activités, présentée aux patients comme « collègue » ou « chercheuse en sociologie ». Ces phases d'observation m'ont permis d'assister à l'ensemble des activités des professionnels et bénévoles : entretiens avec les travailleurs sociaux, consultations médicales, entretiens infirmiers, distribution de médicaments, atelier de prévention, réunions de coordination, réunions d'équipe, permanences dans des structures d'hébergement. Pendant ces observations ou par la suite, j'ai effectué dix entretiens semi-directifs, avec la plupart des membres des équipes (travailleurs sociaux, médecins, infirmiers, secrétaires et membres de la direction), afin de compléter les observations et échanges informels, et recueillir plus précisément les points de vue des professionnels. L'analyse proposée s'appuie principalement sur le matériel ethnographique et historique recueilli ainsi que sur les données d'activité des différents services (rapports d'activité, documents de présentation de projets mis en place, brochures...). 


\section{Le RVH, acteur de première ligne de l'accueil des populations migrantes}

8 Les réseaux de santé sont des dispositifs associatifs qui se sont développés là où le système de santé ne proposait pas une réponse adaptée à des besoins spécifiques ou aux transformations de la demande sociale en matière d'accès aux soins (Poutout, 2005). Ils sont alors apparus dans les interstices du système de santé, ciblant des populations (toxicomanes, personnes âgées...) ou des pathologies (maladies infectieuses chroniques, diabète, cancer...) pour compléter les prises en charge proposées par la médecine libérale et hospitalière (Defert, Cabé, \&Uhalde, 2005). Parmi les premières initiatives, on retrouve les réseaux ville-hôpital (RVH) apparus dans les années 1990 pour répondre au déficit de prise en charge des patients atteints par le virus du sida. Alors que des associations brisent progressivement le silence autour de l'épidémie, des professionnels de santé s'organisent en réseaux locaux sur l'ensemble du territoire national pour accompagner des patients dans l'accès aux soins (Fassin, 1994). Le réseau de santé faisant l'objet de notre étude est l'un d'eux et a gardé son nom, quand bien même ses activités ont largement évolué.

\section{Une structure pionnière dans l'accès aux soins des personnes précaires sur son territoire}

9 Le RVH est aujourd'hui partagé en deux entités fonctionnant de manière autonome, un centre d'accueil et d'accompagnement à la réduction des risques pour usagers de drogues (CAARUD) et un réseau de santé dédié aux personnes en situation de précarité. Si c'est essentiellement l'activité de ce dernier qui nous intéresse, un aperçu de l'histoire de l'ensemble du RVH permet de comprendre la construction de l'action auprès des personnes en situation de précarité sur le territoire :

10 Association de loi 1901, le RVH est créé en 1991 par un groupe de soignants, de travailleurs sociaux et de membres d'associations locales pour améliorer la prise en charge du VIH entre la ville et l'hôpital et construire une certaine expertise autour de cette "nouvelle» pathologie sur le territoire. Alors que l'épidémie concerne localement principalement des usagers de drogues, le réseau développe des actions de formation des professionnels de santé et de prévention auprès des publics à risque. Avec le soutien de l'hôpital, un dispositif expérimental de réduction des risques est mis en place dès 1993 dans les communes alentour et permettra d'obtenir l'agrément de CAARUD en 2006.

11 La mission actuelle de réseau de santé précarité est également le fruit d'une initiative locale, intégrée par le RVH quelques années après sa création. A l'époque, les patients non couverts par l'Assurance maladie doivent présenter des bons d'aide médicale gratuite pour bénéficier d'une consultation, mais la complexité et les dysfonctionnements dans l'obtention de ces bons amènent à d'importants renoncements aux soins. Face aux difficultés de prise en charge des patients en situation précaire, un groupe de médecins du territoire aidés de pharmaciens et travailleurs sociaux, tous bénévoles, mettent en place une consultation gratuite hebdomadaire appelée localement "La Préca ». Très rapidement, la consultation est intégrée au RVH et bénéficie alors du soutien financier de l'hôpital par la mise à disposition de locaux et d'un praticien hospitalier pour sa coordination médicale. Cette 
consultation a perduré dans le temps et constitue encore aujourd'hui une des principales activités du réseau. Dans son format actuel, elle est organisée tel un circuit : elle permet de rassembler en un même moment, c'est-à-dire sur un même lieu et sur un créneau de quelques heures, une évaluation sociale avec un travailleur social, une consultation médicale avec un médecin puis la délivrance de traitements par une infirmière.

12 Alors que les premières initiatives reposaient uniquement sur des bénévoles, le maintien du partenariat avec l'hôpital de secteur, la reconnaissance institutionnelle par l'Agence régionale de santé (ARS) et la multiplication des soutiens budgétaires ont permis de progressivement financer une activité salariée (environ 5 contrats équivalents temps plein au printemps 2018 dédiés aux activités du réseau de santé) et développer d'autres activités auprès des populations précaires. Grâce aux postes de travailleurs sociaux, le réseau effectue des accompagnements à l'ouverture et au maintien des droits à l'Assurance maladie. Depuis 2009, un partenariat avec Pharmacie humanitaire internationale permet la distribution gratuite de médicaments aux personnes ne présentant pas de couverture sociale. En 2016, face à une forte demande, le RVH met en place une consultation dentaire gratuite. Depuis 2015 se développent également des actions "d'aller vers " auprès de campements, bidonvilles et centres d'hébergement social du territoire ainsi que des ateliers sociolinguistiques auprès de femmes immigrées. Une partie des interventions, en particulier le temps médical, repose néanmoins toujours sur un engagement bénévole.

13 L'histoire du $\mathrm{RVH}^{5}$ montre comment celui-ci s'est constitué par des professionnels en réaction à des contextes d'urgence sociale à l'épreuve de dysfonctionnements et cloisonnements du système de santé et s'est progressivement institutionalisé. Pour comprendre comment s'inscrit l'activité du RVH dans le paysage local, il semble alors d'abord nécessaire de décrire ses liens avec ses deux principaux partenaires médicosociaux que sont la PASS et l'EMPP de secteur.

\section{Une action « en réseau » avec la PASS et l'EMPP}

14 Mise en place en 2009, la PASS de secteur proposait d'abord essentiellement des accompagnements sociaux pour l'ouverture des droits à une couverture maladie. En 2016, elle se médicalise à raison d'une journée de consultation hebdomadaire et formalise sa collaboration avec le RVH. Les deux structures ont le même médecin coordinateur. En routine, le service PASS oriente les patients vers la consultation hebdomadaire du réseau, qui vient compléter sa propre offre, et, surtout, pour la récupération de médicaments. Pour la majorité des prescriptions effectuées par le médecin, les patients sont orientés au RVH, munis d'un plan de la ville et d'un «bon PASS » pour récupérer leurs traitements. L'hôpital constitue ainsi le principal orienteur pour l'activité de pharmacie du réseau (environ $60 \%$ des distributions du RVH en $2017)^{6}$. La PASS s'appuie également sur le réseau de médecins bénévoles du réseau pour orienter des patients ayant obtenus leur couverture sociale. De manière réciproque, l'équipe du RVH informe systématiquement les patients de l'existence de la PASS comme autre possibilité de soins gratuits et la fait apparaître dans sa brochure au même plan que ses propres activités. La collaboration entre la PASS et le RVH se joue également dans le partage de pratiques. Par exemple, le médecin coordinateur a décidé 
d'introduire en 2019 un temps infirmier dans le circuit de la consultation de la PASS, sur le même modèle que la consultation du RVH.

15 L'EMPP rattachée au service psychiatrique de l'hôpital a été mise en place en 2008. Elle est composée de deux infirmiers en psychiatrie, un travailleur social, une psychologue et une secrétaire et est supervisée par un psychiatre de l'hôpital. L'équipe effectue des maraudes sur une partie de son secteur, propose des permanences auprès de structures d'hébergements et des entretiens dans ses locaux. Dans sa mission d'interface, elle fait le lien entre les partenaires sociaux et les centres médico-psychologiques pour favoriser des prises en charge et tient une instance de partage d'expérience destinées aux professionnels de l'hébergement social. La collaboration entre le RVH et l'EMPP relève d'orientations réciproques: le RVH adresse des patients vers l'EMPP par l'intermédiaire de fiches d'orientation; réciproquement l'EMPP oriente des patients sans couverture sociale vers la consultation du RVH et sa pharmacie humanitaire. Depuis 2015, cette routine est complétée par l'intervention une semaine sur deux d'un infirmier de l'EMPP à la consultation hebdomadaire du RVH.

La collaboration du RVH avec la PASS et l'EMPP repose sur la mutualisation des pratiques et des moyens de chacun, principalement les consultations gratuites et la distribution de médicaments qui représentent des ressources particulièrement rares. Elle est permise par la proximité spatiale des structures, mais aussi, voire surtout, par l'engagement des professionnels dans leur coordination. En particulier, la réalisation et la distribution de supports d'information et de plans personnalisés permettant d'orienter les patients d'un lieu d'accueil à l'autre semblent indispensables à la visibilité des structures et à leurs collaborations. La dernière partie de cet article revient sur les conditions et moyens de cette coordination.

\section{Aujourd'hui, un dispositif soignant essentiellement des étrangers en situation précaire}

17 L'unique condition, du moins en théorie, pour accéder à la consultation gratuite du RVH est l'absence de protection sociale. L'évolution de la file active de la consultation (fig. 1) montre comment celui-ci reçoit désormais essentiellement des étrangers. Elle indique notamment une forte augmentation de la proportion de demandeurs d'asile, passant d'environ 30\% en 2011 à près de 50\% depuis 2016, alors que ces derniers sont éligibles à la protection universelle maladie (PUMA) et sa complémentaire gratuite (CMU-C). Cette surreprésentation des étrangers en situation précaire, et en particulier des demandeurs d'asile, résulte d'une combinaison de facteurs relatifs à l'évolution des conditions d'accès à une couverture sociale, à l'importance de la population concernée sur le territoire, et à l'évolution de sa visibilité auprès des partenaires et des usagers. En d'autres termes, elle est le produit d'un contexte légal national, d'un contexte démographique local et de la construction d'un réseau de partenaires à différentes échelles. 
Figure 1 : Evolution de la file active de la consultation du RVH de 1997 à 2018

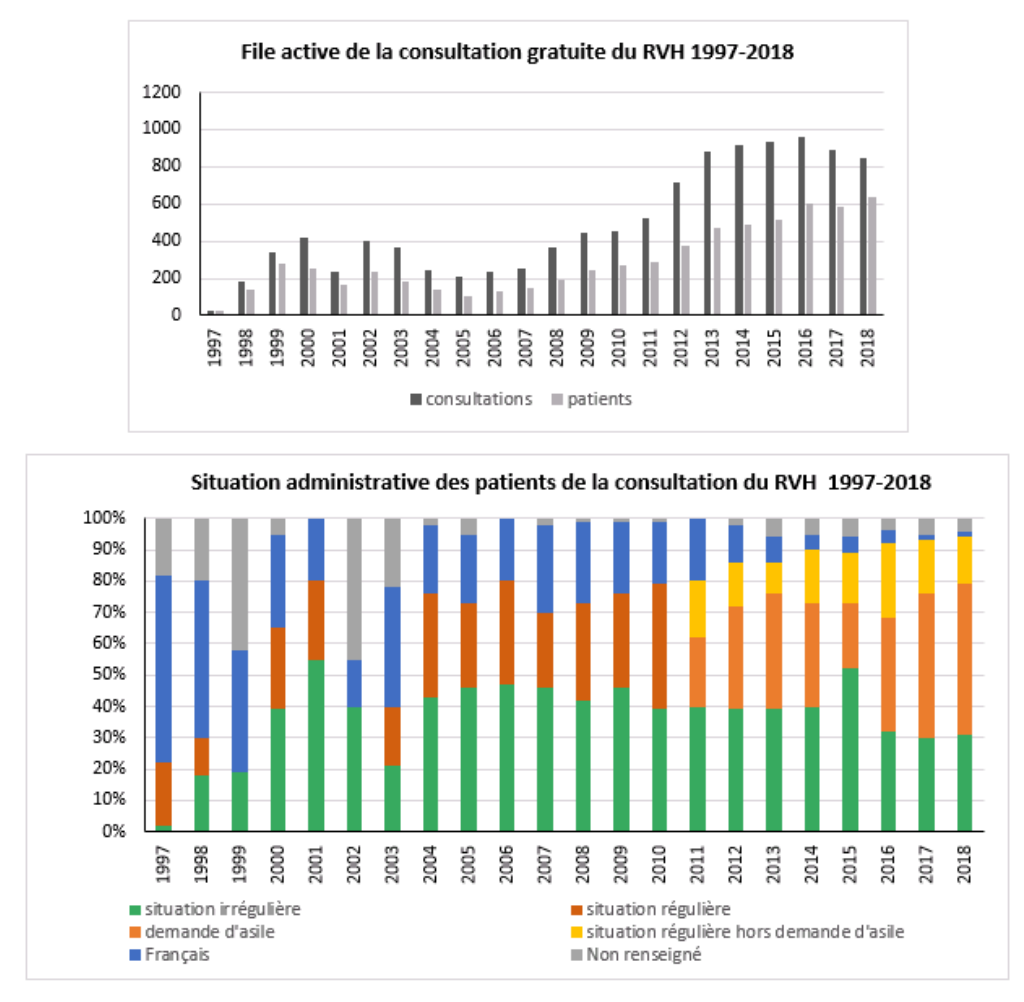

Sources : rapports d'activité 1997-2018

\section{Compenser les difficultés d'accès à une couverture sociale}

18 L'ambition d'une protection sociale universelle est marquée en 1999 par la réforme introduisant la couverture maladie universelle et sa complémentaire gratuite (CMU-C) pour les Français et les étrangers en situation régulière, ainsi que l'Aide médicale d'État (AME) pour les étrangers en situation irrégulière. Ces deux régimes ouvrent alors l'accès à une couverture sociale pour les personnes ne remplissant pas les conditions nécessaires pour être affiliés à un régime de l'Assurance maladie lié à leur activité professionnelle ou étudiante. A ce titre, les chiffres du RVH reflètent dans une certaine mesure un décloisonnement du système de santé de droit commun pour les Français en situation précaire. Même s'ils représentaient encore autour de 15\% jusqu'en 2011, ils représentent depuis 2015 moins de 5\% de la population reçue. La réalité est en revanche plus contrastée pour les étrangers, y compris en situation régulière sur le territoire. Plusieurs facteurs explicatifs coexistent. Plusieurs enquêtes ont relevé d'abord l'importance du non-recours à la CMU-C et l'AME (Chauveaud \& Warin, 2016; Jusot et al., 2019), y compris des personnes exprimant des besoins en santé importants. Elles analysent ce phénomène au prisme d'obstacles administratifs et sociaux que rencontrent particulièrement les étrangers: les documents demandés pour la constitution des demandes peuvent être difficile à fournir, notamment quand il s'agit de justifier d'une absence de ressources, fournir un relevé d'identité bancaire, ou attester d'un hébergement que l'on n'a pas (Carde, 2009 ; Gabarro, 2012). En 2003, un critère de résidence de trois mois pour bénéficier de l'AME est venu exclure les primoarrivants $^{7}$ et suppose pour les autres d'être en mesure de justifier de leur présence sur le territoire. En 2019, ce même critère de résidence a été établi aux demandeurs d'asile 
pour l'accès à la $\mathrm{CMU}-\mathrm{C}$, prolongeant alors la période de non-accès au système de droit commun. De plus, de nombreux étrangers sont amenés à basculer de la CMU-C à l'AME ou inversement au gré de l'évolution de leur situation administrative (lors de l'obtention ou de la fin d'un titre de séjour, lors d'un refus de demande d'asile par exemple), entrainnant des périodes sans couverture sociale. Au-delà de l'obtention des droits, des travaux ont également relevé l'existence de pratiques de traitement différenciés et discriminatoires voire des refus de soins envers les professionnels de santé à l'égard des bénéficiaires de la CMU-C ou de l'AME (Carde, 2007 ; Boisguerin \& Haury, 2008), alimentant le renoncement aux soins ou le recours aux dispositifs de soins dédiés à ceux qui n'ont pas de couverture sociale.

19 Ces délais et obstacles à l'accès à une couverture sociale et plus généralement aux soins permettent de comprendre pourquoi les consultations gratuites à l'image de celle du RVH sont amenés à accueillir des personnes qui devraient normalement avoir accès au système de droit commun, notamment les demandeurs d'asile et les étrangers en situation irrégulière présents depuis plus de trois mois.

\section{Un territoire accueillant de plus en plus de demandeurs d'asile}

20 Au-delà-de la question des droits, l'évolution du contexte démographique local en matière d'accueil social permet de comprendre l'évolution de la file active du RVH, et en particulier la proportion croissante de demandeurs d'asile. L'organisation de l'accueil des demandeurs d'asile sur le territoire français est cadrée par le Dispositif national d'accueil (DNA), qui prévoit notamment l'hébergement de ceux qui en font la demande. Depuis 2015, le contexte d'intensification des flux migratoires vers l'Europe et l'augmentation des demandes d'asile a entraîné une transformation et une extension importante du DNA, marqué notamment par des stratégies de répartition sur le territoire. Dans un objectif «d'équilibre » des demandes sur le territoire français, les lois relatives à l'asile de 2015 et $2018^{8}$ ont consolidé le schéma national d'accueil en y précisant la part précise des demandeurs d'asile pouvant être accueillis dans chaque région. En Île-de-France, cette répartition s'exerce aussi au niveau infrarégional, en témoigne l'augmentation particulière de la capacité d'hébergement de demandeurs d'asile dans les départements de grande couronne. Entre 2016 et 2018, le nombre de places d'hébergement du DNA sur le département étudié a augmenté de $196 \%$ tandis que celui des dispositifs de droit commun augmentait de $48 \%$. Sur le territoire faisant l'objet de cette recherche, cela s'est traduit par l'ouverture entre 2015 et 2017 de deux centres d'hébergement d'urgence migrants (devenus Hébergement d'urgence pour demandeurs d'asile (HUDA) en 2019) et d'un Programme d'accueil et d'hébergement des demandeurs d'asile (PRAHDA). En 2018 se sont ajoutées 150 places d'un centre d'accueil et d'examen des situations (CAES), caractérisé par un accueil très temporaire (théoriquement 11 jours maximum) avant un transfert vers un autre centre. Il suppose alors un renouvellement constant des individus accueillis, essentiellement primoarrivants et pour qui généralement aucune procédure d'accès aux droits n'a été entamée.

21 L'augmentation des capacités d'hébergement de demandeurs d'asile depuis 2015, notamment au sein de dispositifs proposant un accueil de courte durée, a affirmé le territoire (sur lequel se situe la Préfecture) comme un territoire de transit de demandeurs d'asile. Néanmoins la présence d'une population sur un territoire ne suffit 
pas à l'utilisation d'un service par celle-ci. En l'occurrence, le RVH a été mobilisé dès l'ouverture de nouvelles structures d'accueil sur le territoire.

\section{Le RVH mobilisé dans l'accueil des étrangers sur le territoire}

Dès l'ouverture des centres d'hébergement à partir du printemps 2015, le RVH et le service de santé public de l'hôpital ont été sollicités par l'ARS pour effectuer des consultations médicales et des actions de prévention et dépistage au sein des lieux d'hébergement. Ces interventions se sont inscrites dans un ensemble d'actions visant à évaluer l'état de santé des personnes évacuées des campements et mises à l'abri dans des centres d'hébergement ${ }^{10}$. Partout où des personnes sont installées, il s'agit de proposer un premier contact avec le système de santé : recenser les besoins, proposer des orientations et prévenir la transmission de maladies contagieuses particulièrement redoutées sur les campements et les lieux d'accueil collectifs (Chaud et al, 2017). Entre 2015 et 2017, le RVH est ainsi intervenu une vingtaine de fois sur les deux HUDA et le PRAHDA, au rythme des opérations d'évacuations. De même, lors de l'ouverture du CAES en 2018, le médecin coordinateur est désigné par l'ARS pour intervenir sur place puis pour la coordination médicale du CAES.

Au-delà du soutien apporté au moment des arrivées de nouveaux résidents, les interventions dans ces centres dès leur ouverture ont permis aux équipes sociales d'identifier le RVH et la PASS comme les acteurs médico-sociaux de proximité pour y orienter leurs hébergés dont les droits ne sont pas encore ouverts. En 2018, environ $20 \%$ des patients vus au RVH et $30 \%$ des patients reçus à la consultation de la PASS étaient directement adressés par des dispositifs d'hébergement de demandeurs d'asile. De même, le CAES est devenu l'un des principaux orienteurs du réseau en ce qui concerne son activité de pharmacie.

La mobilisation par l'ARS du RVH et de la PASS dans l'accueil de demandeurs d'asile montre comment ces deux acteurs ont été identifiés et mobilisés comme des lieux de traitement, en première ligne, des enjeux d'accès aux soins des étrangers sur le territoire. Dans ce contexte territorial et face aux orientations nationales en matière d'accès aux soins des migrants, il s'agit alors de s'intéresser à la manière dont ces dispositifs ont adapté et transformé leurs activités pour répondre aux besoins et contraintes des populations.

\section{Une évolution des pratiques et des enjeux de coordination pour répondre à la prise en charge des populations migrantes précaires}

L'accueil grandissant d'une population primo-arrivante sur le territoire a conduit le RVH mais aussi la PASS et l'EMPP à développer des dispositifs spécifiques et à s'ouvrir à des partenaires propres à l'accueil des étrangers, en particulier des demandeurs d'asile. Cette spécification de la prise en charge passe par des interventions directement auprès des populations ciblées et la mise en place en interne de consultations spécifiques. Ces pratiques témoignent du développement d'une «problématique migrants» sur le territoire. 


\section{Des interventions sur les lieux d'accueil de demandeurs d'asile}

26 La faible mobilité des populations en centre d'hébergement, parfois très éloignés du réseau de transports en commun et l'importance du non recours aux soins des individus en situation de précarité justifient le développement d'actions directement sur les lieux d'accueil. Les opérations de dépistage et les bilans de santé à l'ouverture des centres d'accueil de demandeurs d'asile s'inscrivent dans cette démarche dite "d'aller-vers", visant à repérer des besoins en matière de santé en évacuant les contraintes d'accessibilité territoriale et fonctionnelle (Ravon et al., 2000 ; Mellier, 2006). Lorsqu'elles s'inscrivent dans un long terme, ces opérations hors-les-murs peuvent prendre la forme de permanences sur les lieux d'accueil, ce que nous avons observé sur le territoire étudié. Depuis 2015, le RVH assure une permanence hebdomadaire à la structure de premier accueil des demandeurs d'asile (SPADA) pour effectuer des ouvertures de droits et orienter des individus en demande de soins vers des structures médico-sociales adaptées. Cette permanence participe à donner une visibilité du réseau auprès d'un public généralement peu informé de leurs droits et des structures existantes sur le territoire. De son côté, l'EMPP assure également depuis 2015 des permanences dans certains CADA et HUDA du territoire, qui permettent aux équipes sociales de proposer un soutien psychologique à des hébergés sans la charge d'accompagner les résidents vers un lieu de soin. Parallèlement, l'équipe mobile a mis en place une instance de discussion et d'échange de pratiques dédiée aux équipes sociales des centres d'hébergement de demandeurs d'asile du territoire, qui est distingué d'un groupe similaire destiné aux autres partenaires sociaux de l'équipe mobile (mairie, foyers, CHRS, associations). De même que les permanences auprès de structures d'accueil représentent des nouvelles portes d'entrée vers le système de santé qui sont spécifiques aux personnes en demande d'asile, cette distinction dans le soutien apporté aux différentes équipes sociales relève d'un effet de ciblage et de différenciation entre les actions auprès des demandeurs d'asile et celles dédiées aux autres publics précaires.

\section{Le déploiement de « consultations migrants »}

La reconnaissance de facteurs de vulnérabilité de migrants en situation de précarité, dans un contexte où le système de santé de droit commun ne présente pas les dispositifs permettant d'y répondre, ont entraîné le développement en France de consultations spécifiques principalement dans deux champs sanitaires: la prise en charge en santé mentale d'une part, la prévention et le dépistage d'autre part (Comitti \& Patureau, 2005). C'est également dans ces deux champs de la santé que nous avons retrouvé la mise en place de consultations dédiées au public migrant sur le territoire.

28 A travers la catégorie du traumatisme, autour de la notion «d'état de stress posttraumatique " $^{11}$ s'est développée à partir des années 1980 une "clinique de l'exil » (Benslamana, 2003) fondée sur la reconnaissance d'effets psychiques de l'expérience migratoire. Observant des prévalences plus élevées de troubles psychiques sur les populations migrantes, des études ont montré comme le départ de son pays sans perspective de retour, les violences vues ou subies avant ou pendant le parcours migratoire mais également la précarité multiforme liée à la condition d'étranger sur le territoire ou l'expérience de l'attente et des procédures administratives favorisent la résurgence d'effets traumatiques (Carswell et al., 2011; Nguyen, 2014; Veïsse et al, 
2017). Beaucoup de ces recherches, ainsi que les initiatives de développer des consultations psychosociales centrées sur les psycho-traumatismes liées à l'exil ont d'abord été portées par des associations qui se sont spécialisées dans l'accueil de personnes migrantes. En milieu hospitalier se sont également développées des consultations transculturelles basées sur des approches ethnopsychiatriques (Crenn, 2000), et à partir de 2005, les EMPP sont devenues des vecteurs de ces approches. Sur le territoire francilien étudié, c'est en 2011 qu'est mise en place une "consultation migrants » par l'EMPP pour répondre à l'importance de la demande. L'EMPP effectue un premier contact, évalue les besoins d'une prise en charge spécifique et propose ou non aux individus l'entrée dans la consultation. Par rapport à son activité de routine, cette consultation représente la possibilité de proposer une prise en charge en plusieurs temps, un suivi en coordination avec un psychiatre de l'hôpital. Elle est perçue, comme le précise une psychologue, comme « un moyen d'aller au-delà du soutien psychologique en proposant davantage une thérapie ".

Concernant la prévention, la réalisation de bilans de santé dans les centres de mise à l'abri lors des évacuations de campements (Guisao et al, 2017), la collaboration des lieux d'accueil avec les centres de lutte anti-turberculeuse et les centres de dépistage ont montré l'importance de la problématique de la prévention sur la manière de mobiliser les intervenants sociaux et les structures d'accueil dans la prévention des maladies infectieuses auprès des publics migrants. Celle-ci occupe une place centrale dans la circulaire de juin 2018, qui revendique l'accès des primo-arrivants à un « rendez-vous santé » devant inclure la délivrance d'informations sur le système de santé, un examen clinique, une évaluation et mise à jour du statut vaccinal, ainsi qu'un ensemble de dépistages. Au RVH et à la PASS ont été mises en place en 2018 et 2019 des consultations répondant étroitement aux objectifs d'un tel rendez-vous. A partir d'un recueil de données socio-démographiques, notamment sur le parcours migratoire, et d'un bilan de santé incluant les pratiques addictives, d'éventuelles violences subies, la sexualité ou encore la nutrition, elles visent à proposer des orientations (par exemple vers une consultation de spécialiste ou vers l'EMPP), mettre à jour certains vaccins et proposer un dépistage des principales IST et de la tuberculose. En pratique, il s'agit de consultations longues (environ $1 \mathrm{~h}$ ), permettant au médecin d'identifier et de répondre à des situations de vulnérabilités liées au parcours migratoire et aux difficultés d'accès aux soins dans le pays d'origine et/ou en France. Au RVH, cette consultation est appelée «Consultation de Prévention Migrants ». A la PASS, la consultation de prévention mise en place a pour spécificité d'être dédiée essentiellement aux mineurs non accompagnés (MNA), répondant à une explosion de la demande en bilans de santé par les partenaires de l'hébergement accueillant des mineurs. Dans ce cas présent, il a été question de mettre en place à une catégorie spécifique de migrant en situation de précarité, les MNA, dont la problématique d'accueil émerge également sur le territoire.

Plusieurs travaux décrivent le déploiement ailleurs sur le territoire français de telles consultations spécifiques et de dispositifs visant à faciliter la prise en charge des populations migrantes, par exemple en Bretagne et en Alsace (Hoyez, 2011 ; Gerbes et al, 2015; Pian, Hoyez \& Tersigni, 2018). Si les initiatives prennent des formes d'organisation variables selon les configurations locales, elles sont à chaque fois portées par des acteurs dédiés à la prise en charge des populations en situation de précarité, c'est à dire essentiellement les EMPP, les PASS ou des associations comme les réseaux de santé. Ces différentes structures sont des lieux de redéfinition et de traitement en première ligne de la «santé des migrants" (Geeraert, 2017). Du 
diagnostic tardif de maladies chroniques à la prise en charge d'un état de stress posttraumatique en passant par une activité de dépistage et de rattrapage vaccinal, ces structures endossent des rôles d'innovateurs voire de laboratoires de l'action médicosociale tournées vers les populations migrantes. Envisagées comme les principales réponses locales aux prérogatives nationales relatives à l'accueil des étrangers (D’Halluin \& Hoyez, 2012; D'Halluin, 2012), elles sont à la fois mobilisées et encouragées par leurs tutelles à développer des actions et des dispositifs orientées vers les populations migrantes arrivant sur leur territoire.

\section{Face à l'errance géographique du public accueilli, la problématique de l'inscription territoriale du réseau d'acteurs}

31 Le public accueilli au RVH, la PASS ou l'EMPP est caractérisé par une forte instabilité résidentielle, de lieux d'hébergement en lieux d'hébergement ou de proches en proches acceptant de les accueillir pour des durées variables. Si les dispositifs spécifiques précités permettent en partie de s'adapter à cette instabilité géographique (en se rendant sur les lieux, en abordant davantage la question des conditions de vie dans les consultations), des situations rencontrées par les professionnels témoignent des difficultés à prendre en compte ces situations d'errance dans la prise en charge des individus et posent la nécessité d'étendre l'inscription territoriale des réseaux de partenaires.

\section{Accueillir des individus domiciliés sur le territoire mais qui n'y vivent pas}

Par opposition à la réalité de leur situation résidentielle, la demande d'asile exige une adresse stable tout au long de la procédure. Dès lors, même s'ils parviennent à être hébergés par un tiers ou un service du 115, la plupart des demandeurs d'asile se font domicilier auprès d'une association ou d'une structure de premier accueil des demandeurs d'asile (SPADA), où ils pourront récupérer du courrier tout au long de leurs démarches. Cette domiciliation contrainte ne correspond alors que très rarement à la commune où les individus vivent au quotidien, d'autant qu'il n'y a qu'une SPADA sur le département. Pour certains, selon les moments de leur parcours, la SPADA peut se trouver à l'autre bout du département voire de la région. Pour le RVH, dont environ un tiers des patients déclarent être orientés par la SPADA ou l'OFII, ce décalage entre le lieu de vie et la domiciliation pose un réel problème d'orientation vers des professionnels de santé appropriés. Lors de la permanence hebdomadaire à la SPADA, l'assistante sociale du RVH est systématiquement confrontée à plusieurs individus hébergés à l'autre bout du département ou dans un autre département, si bien qu'une orientation à la consultation du réseau ou à la PASS semble particulièrement inadaptée. Dépourvue d'un accès à un réel annuaire de partenaires hors du territoire, elle tente alors, à partir de quelques documents glanés sur internet, de donner des informations sur les PASS les plus proches du lieu de vie de l'individu. Dans le souci de répondre le mieux possible à la situation résidentielle des individus, elle tente de manière informelle, d'étendre spatialement ses possibilités d'orientation.

33 Le décalage entre la domiciliation et l'espace de vie est d'autant plus problématique pour l'EMPP qu'elle est censée respecter la sectorisation de la psychiatrie. Celle-ci implique que la plupart des services de santé mentale, y compris les équipes mobiles, sont rattachés à un ou plusieurs secteurs d'intervention. C'est alors l'adresse de 
l'individu qui définit le service supposé le prendre en charge. L'EMPP intervient sur les quatre secteurs de psychiatrie du sud du département. Si ce territoire est déjà bien vaste pour une seule équipe, la sectorisation suppose qu'elle prenne aussi en charge les patients domiciliés sur le territoire (parfois orientés par leur organisme de domiciliation) mais qui n'y vivent pas. En pratique, la prise en charge de ces individus est discutée par l'équipe au cas par cas, afin d'évaluer les difficultés de prise en charge et de suivi. Dans le cas où la prise en charge est jugée compliquée, il faut alors pouvoir orienter la personne dans un service du secteur de vie, qui n'est théoriquement pas censé la prendre en charge. A l'inverse, elle n'est pas censée prendre en charge les individus vivant sur le territoire mais domiciliés ailleurs, mais est pourtant amenée à le faire. La sectorisation de la psychiatrique est ainsi principalement un problème pour l'inclusion dans les soins; plus que pour le suivi, qui devient complexe lorsque les personnes sont amenées à changer de territoire.

\section{Assurer le suivi des personnes envoyées sur un autre territoire}

Evoquées plus haut, les réformes du droit d'asile de 2015 puis 2018 ont confirmé une stratégie de répartition des demandeurs d'asile sur le territoire. En plus de préciser la part des demandeurs pouvant être accueillis dans chaque région, le schéma national d'accueil prévoit désormais que les services de l'OFII puissent orienter un individu dans une autre région que celle où il a effectué sa demande, sous peine de perdre ses droits. Cette politique de répartition accentue l'incertitude résidentielle des demandeurs d'asile par le risque de se voir envoyer dans un territoire inconnu. Le principe des centres d'accueil et d'évaluation des situations (CAES) est la concrétisation de cette stratégie par l'offre d'un accueil très temporaire avant d'être réorienté. Concernant les résidents du CAES, les professionnels des trois structures médico-sociales sont unanimes sur l'impossibilité d'envisager un suivi ni même de coordonner un début de parcours de soins. "On sait qu'il y a de grandes chances qu'ils soient déjà loin le jour du rendez-vous ", explique une assistante sociale si bien que la réponse ne peut rester que ponctuelle. A ce titre, la création des CAES en 2018 semble entrer en contradiction avec la circulaire sur la coordination des parcours de soins des migrants, pourtant introduite la même année.

La question de l'errance s'avère particulièrement problématique pour l'EMPP dont les prises en charges psycho-sociales supposent généralement de s'inscrire sur un temps long. Les professionnels font part d'une forme de résignation à ne pas proposer un suivi mais plutôt un "soutien", pouvant s'inscrire dans une certaine temporalité (2-3 rendez-vous) mais très souvent ponctuel. Pour ceux dont un suivi aurait commencé, dans le cadre de la « consultation migrant » notamment, la forte mobilité résidentielle des patients augmente considérablement les risques de rupture de soins, déjà plus importants pour les publics en situation de précarité. Lorsqu'un patient est envoyé dans un autre lieu d'hébergement, l'enjeu est alors d'assurer la prise en charge par un confrère de cet autre territoire, souvent d'un autre secteur psychiatrique. En pratique, elle oblige les professionnels de santé à trouver un praticien acceptant de prendre le relais de la prise en charge sur un territoire qu'ils connaissent parfois très mal, et dans un contexte de saturation des dispositifs. Les professionnels font part d'un manque de connaissance réciproque et de moyens de coordination avec les réseaux d'acteurs implantés sur d'autres territoires, même au sein d'un même département. 
36 La nécessité de s'adapter à la situation d'errance des demandeurs d'asile pose la question des échelles pertinentes pour coordonner leur prise en charge médico-sociale. A l'impératif d'une coordination locale, à l'échelle des territoires de vie et cohérente avec la répartition des infrastructures de santé sur le territoire, s'ajoute celui d'une coordination à une échelle qui puisse répondre à la mobilité géographique du public accueilli afin d'éviter les interruptions de soins. Dans quelle mesure ces dispositifs sontils alors capables de « dépasser le stade de l'arrangement local » (Poutout, 2005) ? C'est tout l'enjeu du maillage territorial des réseaux de santé et de l'intégration de leur production collective dans des systèmes d'information plus larges (Bourret, 2008).

\section{Quels moyens pour étendre les compétences de coordination?}

37 En pratique, l'insuffisance des budgets pour développer une collaboration médicosociale et le manque de technologies de l'information et de la communication (TIC) à disposition des acteurs en première ligne font reposer l'effort de coordination sur la multiplication des engagements et responsabilités des professionnels.

\section{La contrainte budgétaire}

38 Le financement des activités des structures médico-sociales relève d'un assemblage de différents budgets accordés selon les missions confiées aux structures et la reconnaissance d'initiatives en matière d'accès aux soins. L'enjeu de débloquer des budgets pour des consultations dédiées ou des actions hors-les-murs vise à la valorisation des facteurs qui font prendre du temps et/ou sont spécifiques à l'accueil de population migrantes, comme le fait d'être confronté à des situations administratives complexes, des pathologies inhabituelles ou des difficultés de communication. Mais le financement de ces besoins spécifiques s'avère laborieux. Si les consultations récemment mises en place par les trois structures ont fait l'objet de nouvelles enveloppes budgétaires de la part de l'Agence régionale de santé, le témoignage des professionnels de santé révèlent qu'elles ont été obtenues au terme de négociations complexes. Par exemple, 2019 est la première année où le pôle de psychiatrie obtient un budget pleinement dédié à la « consultation migrants » de l'EMPP alors que celle-ci existe depuis 2011. Jusqu'alors, son existence reposait sur l'engagement de psychiatres en dehors de leur activité principale, limitant fortement des possibilités d'évolution. Au RVH 77, le financement d'une consultation dentaire en 2017 par l'ARS avait finalement pu se faire grâce à un arrangement avec la PASS et un montage financier complexe entre l'hôpital, la Communauté d'agglomération et le Commissariat général à l'égalité des territoires. Quant à la mise en place de la consultation de prévention, elle était, au printemps 2019, organisée sans budget spécifique, en qualité d'expérimentation et dans la perspective d'obtenir un financement l'année suivante. Dans certains cas, les négociations n'aboutissent pas: alors que le développement de la médiation et de l'interprétariat professionnel en santé figure parmi les axes majeurs du Programme régional pour l'accès à la prévention (PRAPS) et les recommandations nationales, le médecin coordinateur de la PASS et du RVH s'est vu refuser en 2019 le financement d'un poste de médiateur en santé. 
39 Pour les professionnels rencontrés, l'insuffisance des budgets accordés dans un contexte de forte augmentation de la demande en soins amène par ailleurs à un paradoxe de leurs missions, celui d'avoir les moyens de coordonner mais non de développer une activité médicale à coordonner. Si le RVH bénéficie d'une mise à disposition par l'hôpital du médecin coordinateur (à hauteur de 0,1 ETP), il n'a pas les moyens de financer le temps médical à la hauteur de l'activité qu'il propose. L'ensemble du temps médical de la consultation d'accès aux soins et aux droits du réseau est assuré par des bénévoles. Cette situation relève de son statut associatif, de l'héritage de l'initiative des années 1990 et d'un choix de la perpétuer, mais elle montre comment le maintien de l'activité du RVH tient à sa capacité à assurer une " médecine à bas coût ", reposant principalement sur l'engagement des acteurs qui la font vivre.

\section{L'insuffisance voire l'absence de technologies de l'information et de la communication}

40 Alors que les systèmes d'information et les technologies de communication sont décrits comme la clé de la mise en réseau des professionnels de santé (Bourret, 2008 ; Plu et al., 2009 ; Robelet, Serré, \& Bourgueil, 2005), ils apparaissent comme particulièrement absents du travail des trois services observés.

Un élément majeur est l'absence de dossier informatisé permettant la circulation des informations sur un patient. L'absence de supports de transmission apparaît comme un gros manque pour les professionnels, non seulement pour les orientations mais aussi au moment des consultations. Comme le souligne un médecin, les professionnels se demandent parfois s'ils ne sont pas en train de prescrire un énième dépistage à l'individu qu'ils ont en face d'eux : "La condition de migrant fait que parfois ils ont déjà eu des examens ailleurs en France ou à l'étranger. Cela me fait douter de l'utilité de redemander la même chose, les mêmes examens. C'est frustrant. Et puis j'hésite souvent à démarrer des prises en charge un peu longue car on ne sait pas si et quand ils vont partir [...] J'aurais aimé qu'ils aient un dossier médical pour permettre un suivi ». Faisant référence à l'errance des patients, il regrette l'absence de dossier permettant de limiter les difficultés que celleci engendre pour la prise en charge puis le suivi. Au-delà du souci de coordonner le parcours de soins, il remet également en question le sens de son travail et l'utilité de l'aide apportée. A défaut d'un dossier informatisé pouvant circuler entre médecins, les professionnels plébiscitent alors l'utilisation d'un carnet de santé. Mais le recours à un carnet fait division notamment car il pose la question du partage de l'information relative à l'état de santé, pour un public dont les documents administratifs circulent entre divers guichets.

L'absence de moyens de suivi est également un problème rencontré par les travailleurs sociaux. Si ce n'est pas le cas à la PASS, les assistantes sociales du RVH n'ont pas accès au logiciel de consultation d'accès aux droits en ligne (CDR) permettant de suivre les démarches d'ouverture de droits. Il est par exemple impossible de voir si une demande a déjà été lancée lorsqu'une nouvelle personne se présente. Les assistantes sociales ici et là n'ont alors pas d'autre solution que se fier aux échanges avec les patients qui ne sont pas toujours capables de restituer les démarches qu'ils ont entamées. De même, à l'issue de chaque ouverture de droits, elles insistent à plusieurs reprises sur ce qu'elles viennent de réaliser afin que les patients soient en mesure d'expliquer à d'autres travailleurs sociaux ou médecins qu'ils ont déjà effectué cette démarche avec une 
structure. Afin de limiter les doublons, qui contribuent à augmenter les délais de réponse et représentent un coût supplémentaire pour l'Assurance maladie, l'absence de CDR demande aux travailleurs sociaux de développer des stratégies elles-mêmes coûteuses en temps et en énergie.

43 Face à l'absence de technologies de partage d'information, la coordination entre la PASS et le RVH se fait alors sur la base de pratiques informelles. C'est ainsi qu'entre deux patients, l'infirmière de la PASS appelle avec son téléphone personnel le RVH afin de planifier une consultation avec un médecin spécialiste bénévole ou pour demander s'il est encore temps d'envoyer un patient chercher des médicaments. Pour les professionnels, ces arrangement informels sont particulièrement importants au regard de la situation précaire des patients. La faible maîtrise de la langue, les contraintes de mobilités (les patients se déplacent essentiellement à pieds ou en transports en commun, même quand ils sont accompagnés) poussent les professionnels de santé à ménager les solutions les plus simples. Ce «bricolage organisationnel» (Schweyer, 2005) adapté au public migrant précaire se retrouve également dans les supports d'information utilisés. Le RVH s'est ainsi confectionné ses propres documents pour présenter ses activités au public accueilli et orienter les patients vers ses différents partenaires (la PASS, le Cegidd, les PMI, les associations...) (fig. 2). Ces documents incluent des informations et adresses utiles, des plans et des itinéraires particulièrement adaptés à la fois au territoire et au public en situation de précarité. Néanmoins ils représentent un travail de réalisation important, "sur mesure », réalisé par l'assistante de direction en plus de ses principales missions. Ces supports sont également le reflet de l'inscription territoriale du réseau, car c'est finalement là où s'arrêtent ces documents que se trouvent les limites des orientations. La nécessité d'étendre les orientations à d'autres territoires, notamment pour les personnes venant de loin, soulève alors la question de la production ou la mise à jour de supports mais semble fortement limitée par l'absence de systèmes d'information partagés. 
Figure 2 : Des supports de communication « faits maison » pour une orientation plus adaptée.

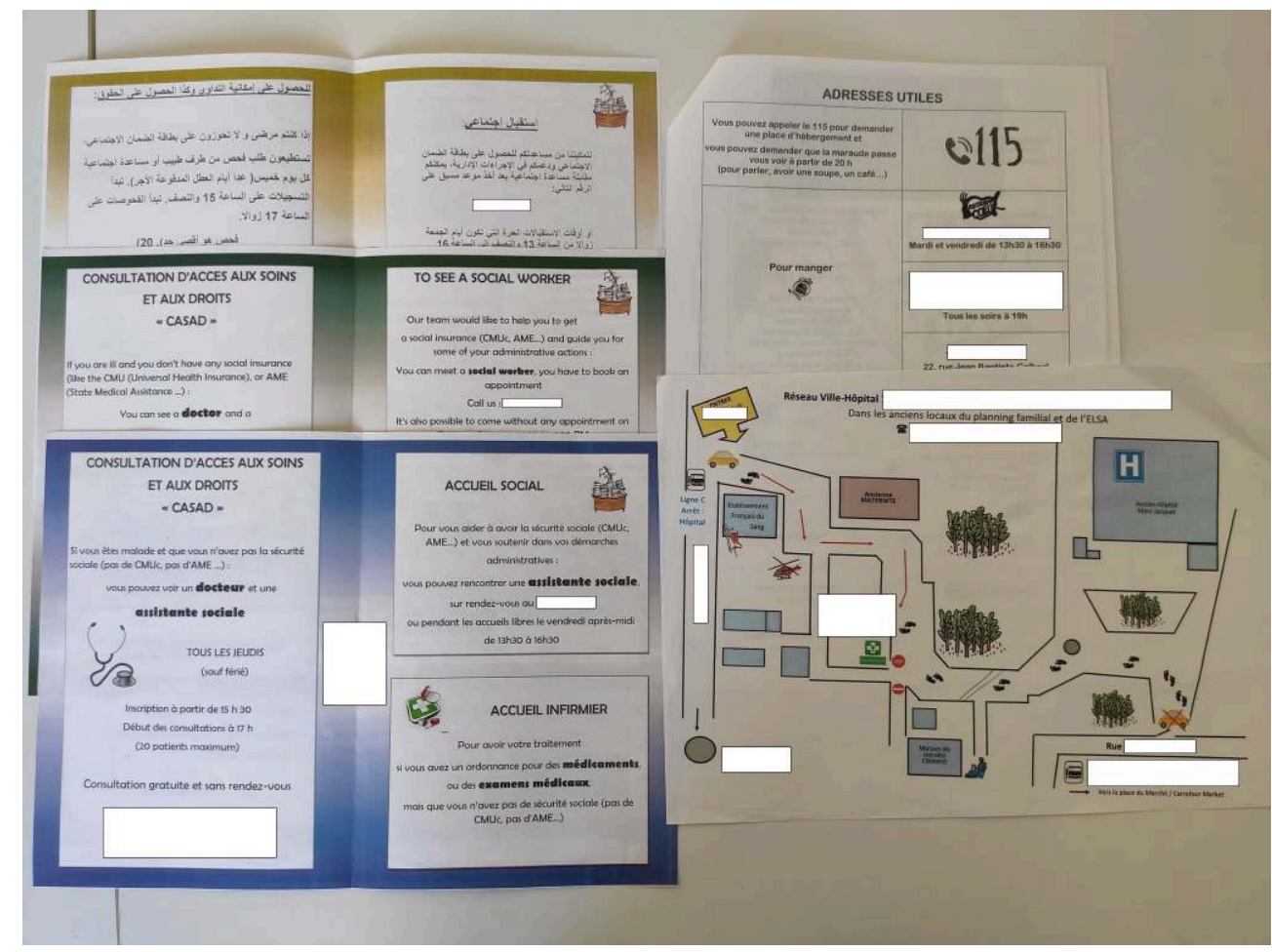

I. Siffert, 2020

L'extension territoriale des réseaux de santé semble ne pouvoir se passer du développement de systèmes d'information, d'autant qu'ils sont également une manière de justifier leur existence auprès de nouveaux acteurs et pérenniser le lien avec ceux qui en font déjà partie (Robelet, Serré, \& Bourgueil, 2005). En outre, à l'image des situations précitées, le manque de technologies de coordination et de communication efficaces contribue à user l'investissement des professionnels de santé et alimente le cercle vicieux d'un sentiment d'isolement de ce type de services, dont l'essence même est pourtant de s'inscrire dans un réseau. Au RVH notamment, la spécificité des dispositifs proposés et l'absence de moyens de coordination avec les autres services s'ajoute à l'isolement spatial (par rapport à l'hôpital notamment) et génère un sentiment de solitude pour répondre aux besoins des patients.

\section{Une coordination qui repose principalement sur la multiplication des casquettes professionnelles}

Le contexte budgétaire et l'absence d'accès aux technologies fait reposer l'effort de coordination sur les professionnels dont les responsabilités ne cessent alors de s'étendre. Cet effet est manifeste au RVH et à la PASS où la plupart des professionnels cumulent plusieurs casquettes. Le médecin coordinateur du RVH est également coordinateur de la PASS et de l'Unité de santé publique (USP), en plus de réaliser des consultations d'infectiologie. Ces différentes responsabilités permettent de faire le lien entre les différents services, comme par exemple faciliter l'accès des patients en situation précaire à des consultations hospitalières ou obtenir plus rapidement des résultats du laboratoire. De même à la PASS, l'infirmière occupe un poste partagé à 50 \% avec l'USP, après avoir travaillé plusieurs années entre le RVH et l'USP. Le format 
partagé de son poste ainsi que son expérience de l'ensemble des services facilitent les orientations des patients d'un service à l'autre. L'organisation observée correspond à un modèle de collaboration très étroite entre infirmière et médecin, dans lequel le médecin reste le coordinateur officiel mais l'infirmière la figure « d'adjointe » (Robelet et al, 2005), forte de son expérience et qui se permettra par exemple d'appeler directement le médecin en consultation pour débloquer des situations compliquées. Néanmoins, le fait d'être partagé entre deux voire trois services entre lesquels une coordination, même informelle, est nécessaire, fait reposer sur une même personne une activité médicale et une activité de coordination sans que celle-ci soit forcément valorisée. La multiplication des casquettes professionnelles évolue alors dans un équilibre social instable, entre un sentiment de montée en compétences, en responsabilité et en autonomie d'un côté et un risque d'épuisement de l'autre.

\section{Conclusion}

A l'instar des prérogatives nationales visant à s'appuyer sur les acteurs médico-sociaux "de proximité " pour la coordination des parcours de santé des étrangers, le RVH, l'EMPP et la PASS que nous avons étudiés sont des lieux d'évolution et d'expérimentation, en première ligne, de la problématique sociale et politique que représente la "santé des migrants" en France. L'analyse de leurs transformations récentes, à travers des partenariats de plus en plus ciblés, des interventions dans les lieux d'accueil des étrangers et la mise en place de consultations dédiées, témoigne d'une prise en charge de plus en plus spécifique des populations étrangères en situation précaire, et notamment des demandeurs d'asile. La prise en compte de l'errance résidentielle dans les suivis et les parcours de soins impose par ailleurs à ces structures de proximité de penser leur construction en réseau à d'autres échelles. Elle invite alors à réfléchir aux dimensions territoriales des inégalités en matière d'accès aux soins, dans un contexte d'une politique de répartition des étrangers sur le territoire. Ces structures médico-sociales portent par ailleurs des stigmates d'un développement dans les interstices du droit commun, dont notamment une certaine carence technologique qui contribue à les isoler encore plus du reste du système de santé. Entre l'informel et l'institutionnel, la coordination de la prise en charge des personnes migrantes repose finalement sur l'engagement et l'énergie d'une poignée de personnes développant des stratégies pour proposer à moindre coût un accès aux soins le plus cohérent possible avec les situations de vulnérabilité des patients. La multiplication de dispositifs de soins labellisés « migrants » interroge également l'adaptabilité du système de santé au public étranger en situation précaire. A rebours d'une volonté de désingulariser la santé des étrangers en France (Fassin, 2009), le souci d'accorder plus de temps au patient en situation de vulnérabilité ou de proposer un interprétariat au patient francophone semble ne pouvoir se passer de dispositifs spécifiques. La tendance à cette spécialisation de certains services pour les étrangers en situation précaire présente alors le risque d'une part d'invisibiliser encore davantage les étrangers dans le système de santé de droit commun, d'autre part d'éloigner encore plus ces services dédiés aux populations précaires du reste du système de santé. Par ailleurs, il semble nécessaire de toujours garder à l'esprit que le recours aux soins des migrants est loin de répondre uniquement à des logiques d'adressage et de "parcours" et s'inscrivent dans un ensemble de facteurs sociaux et spatiaux, dont les contraintes résidentielles et l'accessibilité des structures de santé (Fleuret \& Sécher, 2006 ; Hoyez, 2012). Dès lors, 
notre réflexion invite à décrire plus précisément d'une part les trajectoires de soins des individus, notamment au prisme de leurs trajectoires administrative et résidentielle; d'autre part les relations des acteurs de l'hébergement social avec les dispositifs médico-sociaux et leurs capacités à identifier les ressources en matière de soins et à orienter les personnes qu'ils accueillent.

\section{BIBLIOGRAPHIE}

Agence régionale de santé d'île-de-France (2018), Projet régional de santé 2013-2017 : bilan du programme régional d'accès à la prévention et aux soins (PRAPS)

Agier, M. (2016). Les migrants et nous : Comprendre Babel. Paris : C.N.R.S. Editions.

Beauchemin, C., \& Ichou, M. (Éd.). (2016). Au-delà de la crise des migrants : Décentrer le regard. Paris : Éditions Karthala.

Benslamana, F., (2003), Qu'est-ce qu'une clinique de l'exil ? L'Evolution psychiatrique, 69.

Berchet, C. et Jusot, F. (2010). "Inégalités de santé liées à l'immigration et capital social : une analyse en décomposition." Revue d'Economie Publique, n²4-25, 73-100. URL : http://

journals.openedition.org/economiepublique/8484 ; DOI:10.4000/economiepublique.8484

Boisguerin, B., Haury, B. (2008)., Les bénéficiaires de l'AME au contact avec le système de soins, Drees, Etudes et Résultats, ${ }^{\circ}$ 645. er645.pdf

Bourret, C. (2008), Les réseaux de santé ou la rencontre de la santé et des TIC pour décloisonner le système de santé français, tic \& société, $n^{\circ} 2(1)$.

Carde, E., (2007)., Les discriminations selon l'origine dans l'accès aux soins, Santé Publique, 19, 99-109 DOI:10.3917/spub.072.0099

Carde, E. (2009). Quinze ans de réforme de l'accès à une couverture maladie des sans-papiers : De l'Aide sociale aux politiques d'immigration. Mouvements, $n^{\circ}$ 59(3), 143-156. DOI:10.3917/mouv. 059.0143

Carswell, K., Blackburn, P., Barker, C. (2011), The relationship between trauma, post-migration problems and the psycho-logical well-being of refugees and asylum seekers. International Journal of Social Psychiatry, 57, 107-19. DOI:10.1177/0020764009105699

Chambon, N., \& Le Goff, G. (2016), Enjeux et controverses de la prise en charge des migrants précaires en psychiatrie, Revue française des affaires sociales, 6, 123-140 [texte intégral]

Chappuis, M. (2012). Accès aux soins : Le parcours du combattant des étrangers en situation précaire. Apres-demain, $\mathrm{N}^{\circ}$ 23, NF (3), 24-26. DOI:10.3917/apdem.023.0024

Chaud, P., Haeghebaert, S., Leduc, G., Merlin, B., Janssens, M., Denoual, A., Pascal, M., Simon, N., El Mouden, M., Battist F., Benoit Raeckelboom, B., Souyris, F., Wyndels, K., (2017), Surveillance des maladies infectieuses dans les populations migrantes accueillies à Calais et à Grande Synthe, Novembre 2015 - octobre 2016, Bulletin Epidemiologique Hebdomadaire, $\mathrm{n}^{\circ} 19-20$. [texte intégral] 
Chauveaud, C., \& Warin, P. (2016), Le non-recours à la Couverture maladie universelle Complémentaire. Enquête auprès des populations précaires, Observatoire des non-recours aux droits et services (Odenore), 66 p. [texte intégral]

Cognet, M., Hamel, C., Moisy, M., (2012), Santé des migrants en France : l'effet des discriminations liées à l'origine et au sexe, Revue Européenne des Migrations Internationales, 28 (2), 11-34. DOI: 10.4000/remi.5863

Comité consultatif national d'éthique (CCNE) pour les sciences de la vie et de la santé, (2017), Santé des migrants et exigence éthique, avis $n^{\circ} 127$, Paris.

Comiti, V.-P., \& Patureau, J. (2005), La santé des migrants en France : spécificités, dispositifs et politiques sanitaires, Santé, Société et Solidarité, 1, 129-137. DOI:10.3406/oss.2005.1033

Crenn, C., (2000) Une consultation pour les migrants à l'hôpital, Hommes \& Migrations, 1225, 39-45. DOI:10.3406/homig.2000.3509

Defert, D., Cabé, M.-H., \& Uhalde, M. (2005). Innovation militante et réseau. Sociologies pratiques, $\mathrm{n}^{\circ}$ 11(2), 7-12. DOI:10.3917/sopr.011.0007

Desgrées du Loû, A. (dir.) (2017), Parcours. Parcours de vie et de santé des Africains immigrés en France, La Découverte, 359 p.

D'Halluin, E., (2009), La santé mentale des demandeurs d'asile, Hommes et Migrations, $\mathrm{N}^{\circ} 1282$.

D'Halluin, E., (2012), Les épreuves de l'asile. Associations et réfugiés face aux politiques du soupçon, EHESS, 2012, $302 \mathrm{p}$.

D’Halluin, E., \& Hoyez, A.-C. (2012), L'initiative associative et les reconfigurations locales des dispositifs d'accès aux soins pour les migrants primo-arrivants, Humanitaire, 33.

Drouot, N., Tomasino, A., Pauti M.-D., Corty J.-F., Luminet, B., Fahet, G., Cayla, F. (2012), L'accès aux soins des migrants en situation précaire, à partir des données de l'Observatoire de Médecins du Monde : constats en 2010 et tendances principales depuis 2000, Bulletin épidémiologique hebdomadaire, 2-3-4, 41-44.

Fassin, D., (1994), Les réseaux ville-hôpital, agitateurs depuis 1985. Une approche localiste de la prise en charge des malades du sida, Prévenir, 27, 117-124

Fassin, D. (dir.), (2000), Dossier : Santé, le traitement de la différence, Hommes et Migrations, 1225, $165 \mathrm{p}$.

Fassin, D. (2009). Le droit d'avoir des droits. Hommes \& migrations. Revue française de référence sur les dynamiques migratoires, (1282), 20-23.

Fleuret, S. \& Séchet, R., (2006), Spatialité des enjeux de pouvoir et des inégalités : pour une géographie sociale de la santé, in Penser et faire la géographie sociale. Contributions à une épistémologie de la géographie sociale. Rennes, 333-349.

Fonds d'intervention régional (2018), rapport d'activité 2017, Secrétariat général du ministère des affaires sociales.

Gabarro, C. (2012). Les demandeurs de l'aide médicale d'État pris entre productivisme et gestion spécifique. Revue européenne des migrations internationales, 28(vol. 28-n²), 35-56.

Geeraert, J. (2017). Les frontières à géométrie variable de la citoyenneté. Accès aux soins des migrants dans un dispositif d'assistance. Trajectoires. Travaux des jeunes chercheurs du CIERA, (Horssérie 3). 
Georges-Tarragano, C., Grassineau, D., Astre, H., \& Gaillard, J. (2017). Soigner les migrants dans les PASS. Laennec, Tome 65(1), 25-37.

Gerbes, A., Leroy, H., Leferrand, P., Michel, D., Jarno, P. \& Chapplain, J. (2015). Mieux repérer la souffrance psychique des patients migrants primo-arrivants en consultation de médecine générale et limiter les ruptures de suivis psychiatriques. L'information psychiatrique, volume 91(3), 243-254.

Guisao, A., Jangal, C., Quéré, M., Laporte, A., Riou, F. (2017). La santé des migrants primo-arrivants : résultats des bilans infirmiers réalisés d'octobre 2015 à mars 2016 dans des centres franciliens hébergeant des migrants évacués des campements parisiens, Bulletin Epidémiologique Hebdomadaire, $\mathrm{n}^{\circ} 19-20$

Hervé, C. (2007). Prise en charge médicale et sociale des corps meurtris et des esprits vulnérables. Expérience d'un réseau de santé Asdes, in Boëtsch, G., Hervé, C., Rozenberg, J., (dir.), Corps normalisé, corps stigmatisé, corps racialisé, De Boeck Supérieur "Hors collection, 233-247.

Hoyez, A.-C. (2011). L'accès aux soins des migrants en France et la « culture de l'initiative locale ». Une analyse des contextes locaux à l'épreuve des orientations nationales. Cybergeo : European Journal of Geography.

Hoyez, A.-C. (2012). Mobilités et accès aux soins des migrants en France, Géoconfluences

Hoyez, A.-C. (2015). Territoires, mobilités et santé : Comment composer et penser une offre de soins pour les populations migrantes en situation précaire? Revue francophone sur la Santé et les Territoires.

Jusot, F., Dourgnon, P., Wittwer, J., Sarhiri, J., (2019) Le recours à l'Aide médicale de l'Etat des personnes en situation irrégulière en France : premiers enseignements de l'enquête Premiers pas, Questions d'économie de la Santé, n²43-245.

Lot, F., Antoine, D., Pioche, C., Larsen, C., Che, D., Cazein, F., Semaille, C., Saura, C. (2012), Trois pathologies infectieuses fréquemment rencontrées chez les migrants en France : le VIH, la tuberculose, l'hépatite B, Bulletin épidémiologique hebdomadaire, 2-3-4, 25-30.

Marques, A. (2013). Des équipes mobiles de psychiatrie-précarité : Une forme d'articulation entre les champs social et psychiatrique. Le sociographe, 42(2), 67-77.

Maille, D., \& Toullier, A. (2009). Les dix ans de la CMU. Hommes \& Migrations, (1282), 24.

Médecins du Monde (2018), Rapport de l'Observatoire de l'accès aux droits et aux soins en France en 2017.

Mellier, D. (2006). Précarité psychique et dispositifs d'intervention clinique. Pratiques Psychologiques, 12(2), 145-155.

Mizrahi, A., \& Mizrahi, A., Waits, S., (1993), Accès aux soins et état de santé des populations immigrées en France, CREDES, Paris.

Mizrahi, A., \& Mizrahi, A. (2000). Les étrangers dans les consultations des centres de soins gratuits. Hommes et Migrations, 1225(1), 94-100.

Nguyen, A. (2014). L'agonie administrative des exilés. L'Autre, 15(2), 197-206.

Parizot, I., Chauvin, P. (2003), Le recours aux soins des personnes en situation précaire : une recherche auprès de consultants de centres de soins gratuits en région parisienne, Revue d'Epidémiologie et de Santé Publique, Vol 51, Nº 6 pp. 577-588.

Pian, A., Hoyez, A. \& Tersigni, S. (2018)...... 
Platon, J., \& Lamouche, C. (2012). La permanence d'accès aux soins de santé : Une référence de prise en charge médico-sociale in, Bréchat, P-H., Lebas, J., Innover contre les inégalités de santé. Presses de l'EHESP, $512 \mathrm{p}$.

Plu, I., Gignon, M., Emery, S., Purssell-François, I., Moutel, G. \& Hervé, C. (2009), Modalités et enjeux de la communication externe des réseaux de santé : l'expérience d'un réseau d'accès aux soins, Santé publique, 21 (2), 173-181.

Poutout, G. (2005). Réseaux de santé : Créer du lien pour donner du sens. Sociologies pratiques, $\mathrm{n}^{\circ}$ 11(2), 33-54.

Ravon B., Pichon P., Laval C., Franguiadakis S. (2000), "Aller à la rencontre" : l'engagement des professionnels et bénévoles de l'aide auprès des "personnes en souffrance"., in Micoud A. et Peroni M., Ce qui nous relie, Editions de l'Aube, 71-87.

Robelet, M., Serré, M., \& Bourgueil, Y. (2005). La coordination dans les réseaux de santé : Entre logiques gestionnaires et dynamiques professionnelles. Revue française des affaires sociales, (1), 231-260.

Schweyer, F.-X. (2005), Le travail en réseau : un consensus ambigu et un manque d'outils, Sociologies pratiques, $\mathrm{n}^{\circ} 11(2), 89-104$.

Taieb, A., Taieb, L., \& Vignier, N., (coord.), (2018), La consultation de la solidarité, Prem'édit, 246 p. Tapié de Céleyran, F., Astre, H., Aras, N., Grassineau, D., Saint-Val, T., Vignier, N., Toolan, M., Flicoteaux, R., Aparicio, C., \& Georges-Tarragano, C. (2017). Etude nationale sur les caractéristiques des personnes migrantes consultant dans les permanences d'accès aux soins de santé en France en 2016, in Lot, F., Quelet, S., (dir.), La santé et l'accès aux soins des migrants : un enjeu de santé publique, Bulletin épidémiologique hebdomadaire, $\mathrm{n}^{\circ}$. 19-20, 396-405.

\section{NOTES}

1. Loi $n^{\circ} 98-657 \mathrm{du} 29$ juillet 1998 d'orientation relative à la lutte contre les exclusions.

2. Circulaire DHOS/O2/DGS/6C/DGAS/1A/1B no 2005-521 du 23 novembre 2005 relative à la prise en charge des besoins en santé mentale des personnes en situation de précarité et d'exclusion et à la mise en oeuvre d'équipes mobiles spécialisées en psychiatrie.

3. Si elle ne représente pas le début de l'intensification des flux migratoires vers l'Europe (plutôt identifiée à partir de 2012), 2015 apparait comme une année tournant dans la visibilisation du phénomène et les réponses apportées par les nations européennes. Érigée en symbole, la photo d'Aylan, enfant syrien retrouvé mort sur une plage turque, provoque une émotion à l'échelle mondiale. Plusieurs Etats européens, en particulier la Grèce, l'Allemagne, l'Italie et la France, sont marqués par la mise en visibilité d'une crise de l'accueil des «migrants» sur leur territoire. En France, le nombre de demandes d'asile auprès de l'OFPRA dépasse pour la première fois le seuil des 80000 demandes (rapport de l'OFPRA 2015). On assiste à la médiatisation massive des campements établis en Calaisie et à Paris et de leur gestion par les pouvoirs publics. Entre mai 2015 et novembre 2016 sont enregistrées 31 opérations de mise à l'abri de personnes installées sur des campements parisiens, parallèlement au démantèlement de la «jungle» de Calais. En outre, l'année 2015 est marquée par une évolution importante $\mathrm{du}$ cadre législatif encadrant l'asile: le 22 juillet, la circulaire 
interministérielle « Répondre au défi des migrations », pose les premiers jalons de la loi du 29 juillet 2015 relative à la réforme du droit d'asile.

4. Instruction $n^{\circ}$ DGS/SP1/DGOS/SDR4/DSS/SD2/DGCS/2018/143 du 8 juin 2018 relative à la mise en place du parcours de santé des migrants primo-arrivants, Ministère des solidarités et de la santé.

5. A l'occasion des 20 ans de la consultation d'accès aux droits et aux soins, un ouvrage collectif a été publié. Il retrace l'histoire du RVH et recueille les témoignages des professionnels et bénévoles ayant contribué à le faire évoluer. Taieb A., Taieb L., Vignier N. (coord.), La consultation de la solidarité, Prem'édit, 2018.

6. Données issues des rapports d'activité de la PASS et du RVH.

7. Article 97 de la loi n 2003-1312 du 30 décembre 2003 de Finances rectificative.

8. Loi du 29 juillet 2015 relative à la réforme du droit d'asile puis loi du 10 septembre 2018 pour une immigration maîtrisée, un droit d'asile effectif et une intégration réussie.

9. Le nombre de places en CADA + PRAHDA + CHUM/HUDA + CAES est passé de 824 à 2441 entre décembre 2016 et décembre 2018. Le nombre de places en CHRS + CHU + HUAS est passé de 1663 à 2454 entre décembre 2016 et décembre 2018. Sources : Socle de données hébergement et logement adapté / traitement DRIHL-SOEE / 31 décembre 2018 ; Socle de données hébergement et logement adapté / traitement DRIHL-SOEE / 31 décembre 2016.

10. En 2016-2017, l'ARS missionne le Samusocial de Paris et la Croix rouge française pour effectuer des bilans de santé dans une trentaine de centres d'hébergement d'urgence migrants ouvrant leurs portes dans toute l'île-de-France (Guisao et al, 2017).

11. L'état de stress post-traumatique est un ensemble de symptômes se développant suite à l'exposition à un ou plusieurs événements traumatiques (violences, expositions à la mort...). Les principaux symptômes relevés par les critères du DSM-5 sont la reviviscence et l'évitement de souvenirs traumatiques, des altérations négatives dans les cognitions et l'humeur, une hyper-réactivité.

\section{RÉSUMÉS}

A partir de l'étude d'un réseau de santé précarité et de ses deux principaux partenaires installés en grande couronne francilienne, cet article se propose de réfléchir aux enjeux de coordination que pose la prise en charge médico-sociale des populations migrantes. Il analyse comment se développent dans ces structures dédiées aux populations précaires, des consultations spécifiques et des partenariats propres à l'accueil des étrangers, en particulier des demandeurs d'asile. Alors que l'errance résidentielle du public accueilli appelle à une inscription de la coordination des parcours de soins à de nouvelles échelles territoriales, il interroge les moyens et technologies à disposition des structures pour étendre leurs compétences de coordination.

Although the mechanisms underpinning health inequalities associated with migration are increasingly well described by social epidemiology and health sociology, the operations of health 
care systems involved in the assistance of migrant populations in precarious situations remain largely unexplored.

Based on the study of a precariousness and healthcare network and its two main partner institutions located within the greater Paris region, this article exposes the coordination challenges raised by the medical and social care of migrant populations. This work further analyses the implementation of care activities targeted at migrant populations, and particularly asylum seekers, in these structures already dedicated to precarious populations. These observations highlight how the residential instability of patients seems to call for the coordination of care pathways at new territorial scales. Consequently, we examine the means and technologies available to health facilities to extend their coordination skills in light of such challenges.

In the first part, this article looks back at the evolution of the health network's activity and its collaboration with its two main local partners: the hospital service for free health care (PASS) and the precarity and psychiatry mobile team (EMPP). This health network was created in the 1990s in response to the difficulties encountered by people in precarious situations in accessing healthcare. The evolution of its active patient queue shows a transition towards the reception of mainly migrant populations and particularly asylum seekers (see figure). This tendency can be analysed in light of the administrative barriers in accessing social coverage for irregular migrants and asylum seekers on the one hand, and in the light of the local demographic context on the other. Indeed, as part of the government's strategy for the territorial distribution of asylum seekers enforced since 2015, this area of the Greater Paris region is characterized by the opening of a structure dedicated to initial reception of asylum seekers (SPADA) and the creation of a large number of accommodation places. These structures on the territory generate a new demand in terms of medical and social care, which is directly faced by stakeholders dedicated to precariousness. The mobilisation of the network by the regional health agency at the opening of accommodation centres also explains how it was very quickly identified as a resource structure by social housing partners.

In a second part, this study analyses the emergence of medical and social care operations dedicated to migrant populations. In particular, we describe the increased number of interventions directly in accommodation or reception centres for asylum seekers, and the setting up of so-called "migrant" consultations in the field of prevention and mental health. The goal of these interventions is to focus on physical and psychological vulnerabilities linked to the experience of migration. Therefore, such actions represent entry points to care systems that are specific to migrant populations in precarious situations. In the same section, we explore the difficulties encountered by professionals faced with the residential instability of the population. How to assist someone who actually lives far from the territory where he or she is seeking care? How to ensure the medical follow-up of a person who is sent to another territory by the asylum procedure? The issue of continuity of care obliges local health structures to extend the territorial registration of their partner networks.

Finally, this work analyses the difficulties of overcoming coordination at new territorial scales. In practice, the lack of budget for developing medical and social collaboration and the lack of information and communication technologies mean that coordination efforts are mainly based on the commitment of professionals. It appears that structures dedicated to precarious populations bear the stigma of a development in the interstices of common law.

\section{INDEX}

Index géographique : île de France 
AUTEUR

\section{ISABELLE SIFFERT}

Doctorante en géographie de la santé, Université de Rennes 2, laboratoire Espaces et Sociétés ESO-Rennes (UMR 6590) 